\title{
ANÁLISE DA INTERFERÊNCIA DO ACOPLAMENTO ELETROMAGNÉTICO NA INTERPRETAÇÃO DE DADOS DE POLARIZAÇÃO INDUZIDA E RESISTIVIDADE.
}

\author{
${ }^{1}$ Leonardo da Silva Ribeiro Mocitaiba*, ${ }^{2}$ Edson E. S. Sampaio; ${ }^{2}$ Olivar A. L. de Lima. \\ ${ }^{1}$ Instituto de Geociências da Universidade Federal da Bahia \\ ${ }^{2}$ Centro de Pesquisa e Posgraduação em Geofísica da Universidade Federal da Bahia
}

Copyright 2014, SBGf - Sociedade Brasileira de Geofísica.

Este texto foi preparado para a apresentação no VI Simpósio Brasileiro de Geofísica Porto Alegre, 14 a 16 de outubro de 2014. Seu conteúdo foi revisado pelo Comite Técnico do VI SimBGf, mas não necessariamente representa a opinão da SBGf ou de seus associados. É proibida a reprodução total ou parcial deste material para propósitos comerciais sem prévia autorização da SBGt.

\begin{abstract}
The time variation of a source of alternating current produces an electromagnetic coupling between the transmitting and receiving systems in induced electrical polarization and resistivity (IP/ER) surveys that employ horizontal electrical sounding (HES). This coupling may affect in a sensible way the resistivity and chargeability values and, consequently, modify the interpretation of the data. Millett (1967) constitutes one rare exception of the analysis of the role of this electromagnetic coupling, but evaluated its effect for collinear dipoles in the frequency domain above a homogeneous half-space. This work has extended the analysis to the time domain for both a homogeneous and an n-layered earth. Based on this analysis, it has evaluated the theoretical result of the variation of the voltage and compared it to the observed data from a IP/ER survey with HES, which employed the dipole-dipole array for two dipole lengths: $50 \mathrm{~m}$ and $100 \mathrm{~m}$, and two periods of the square wave of the source current: $4 \mathrm{~s}$ and $16 \mathrm{~s}$. The comparative study of the voltage decay curves made it possible to evaluate the contribution of the electromagnetic coupling to the values of the chargeability and apparent resistivity as a function of the dipole length and the period of the source current. Besides that, the respective pseudo-sections of Haloff have delineated the horizontal and vertical variations of those two electrical properties, providing the interpretation of the geoelectrical section. Therefore, this research is important for the oil industry because it may furnish information that help to: (i) define the structure of the sedimentary strata; (ii) estimate the clay content of the sandstones; and (iii) detect the possible presence of pyrite and pyrrhotite halos at fractured zones of the sediments above the oil reservoir. Furthermore, this research is readily adaptable to electric well logs.
\end{abstract}

\section{Introdução}

Diversas fontes de ruído, naturais e artificiais, atuam em levantamentos conjuntos de polarização elétrica induzida e resistividade (IP/ER). Os ruídos alteram, em maior ou menor grau, o valor das medidas e prejudicam, consequentemente, a modelagem e a interpretação dos dados. O acoplamento eletromagnético destaca-se entre os ruídos artificiais, por ser um componente do dado gerado por qualquer sistema de transmissão e de aquisição cuja fonte de corrente varie com o tempo.

A impedância mútua, $\mathscr{Z}(\omega)$, que representa a razão entre a voltagem no receptor e a corrente no transmissor no domínio da frequência, é a grandeza calculada em levantamentos de IP/ER. Ela depende da variação da resistividade elétrica e da polarizabilidade do subsolo. Entretanto, a análise dos dados de resistividade elétrica baseia-se na função resistividade aparente e pressupõe uma condição de fonte de corrente contínua (DC). Consequentemente, emprega uma impedância invariante com a frequência para formular aquela função. Como a corrente e a voltagem medida variam no tempo, esse pressuposto produz um erro.

A análise dos dados da polarizabilidade constitui um verdadeiro faz-de-conta, pois formula os parâmetros desse fenômeno como se ele ocorresse em uma condição de DC, mas o mensura para diversos valores de frequẽncia (domínio da frequência) ou descreve seu comportamento transiente (domínio do tempo). A causa do fenômeno de polarização induzida nas rochas reside em mecanismos microscópicos capacitivos e de difusão nas interfaces entre a solução eletrolítica e grãos minerais, enquanto o acoplamento eletromagnético é puramente indutivo. Como a ação de ambos resulta em uma impedância variável no tempo ou na frequência, é preciso extrair a contribuição do acoplamento eletromagnético das medidas para obter os dados de IP livres desse tipo de ruído.

\section{Metodologia/Problema Investigado}

Modelos de fonte dipolar horizontal de corrente elétrica constituem a base de diversos métodos geofísicos. Os quatro principais métodos são: (i) GPR (ground penetrating radar) que opera na faixa de frequências de rádio; (ii) SBL (sea bed logging) que opera na faixa de frequências subáudio; (iii) sistemas CSEM's (controlled source electromagnetic) terrestres de bobinas horizontais de forma quadrada ou retangular; e (iv) sistemas terrestres bipolo-bipolo de polarização elétrica induzida e resistividade. O modelo é, aproximadamente, dipolar no caso do GPR. Nos demais, o modelo empregado é a linha de dipolos elétricos, obtida por integração do dipolo elementar ao longo do comprimento da linha.

Um dos objetivos do presente trabalho é determinar a impedância mútua entre os bipolos de corrente e de potencial a partir do acoplamento eletromagnético entre os dois. A base do desenvolvimento algébrico está descrita em Ward (1967) e Wait (1982) para o domínio da frequência. Esse trabalho contém toda a formulação 
algébrica adicional necessária para modelar, no domínio do tempo, a interação entre um modelo de fonte do tipo (iv) e uma terra isotrópica e não magnética formada por camadas horizontais.

\section{Análise da Corrente Elétrica}

A forma de onda (Figura 1) usada nesse trabalho se comporta de maneira periódica, com cada período expresso pela mesma função genérica de tempo. Assim, é possível descrevê-la como uma série de Fourier. Os parâmetros que definem a função são:

$$
I(t)= \begin{cases}0 & \text { para }-T / 2 \leq t \leq-3 T / 8 \\ -I_{0} & \text { para }-3 T / 8 \leq t \leq-T / 8 \\ 0 & \text { para }-T / 8 \leq t \leq+T / 8 \\ +I_{0} & \text { para }+T / 8 \leq t \leq+3 T / 8 \\ 0 & \text { para }+3 T / 8 \leq t \leq+T / 2\end{cases}
$$

,onde $I(\mathrm{t})$ é uma função periódica de período $T$.

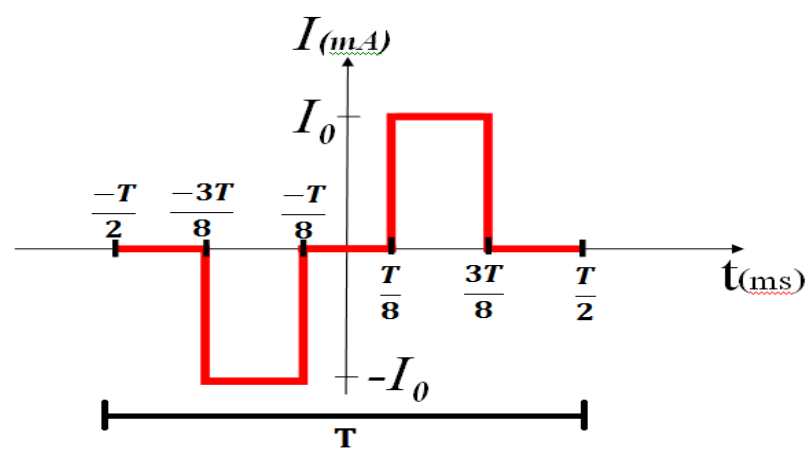

Figure 1: Forma de onda

Com relação à série de Fourier, como a função é impar ela pode ser escrita como:

$$
I(t)=\sum_{j=1}^{\infty} c_{j} \sin \left(j \omega_{0} t\right)
$$

onde,

$$
c_{j}=\frac{4 I_{0}}{\pi j} \sin \left(\frac{\pi j}{2}\right) \sin \left(\frac{\pi j}{4}\right)= \begin{cases}0 & \text { para j par; } \\ \pm \frac{2 \sqrt{2} I_{0}}{\pi j} & \text { para j impar. }\end{cases}
$$

Aplicando a transformada de Fourier, na equação (1), temos a formulação da corrente no domínio da frequência:

$$
I(\omega)=-i \pi \sum_{j=1}^{\infty} c_{j}\left(\delta\left(\omega-j \omega_{0}\right)-\delta\left(\omega+j \omega_{0}\right)\right)
$$

\section{Potencial Elétrico}

Dado um dipolo de corrente elétrica no domínio da frequência, $I(\omega) d x_{0} \hat{x}$, localizado em $\left(x_{0}, y_{0}, z_{0}\right)$, desejamos determinar os campos elétrico horizontal e magnético vertical, $\mathrm{E}\left(x, x_{0}, y, y_{0}, z, z_{0}, \omega\right) \hat{\mathrm{x}}$ e $\mathrm{B}\left(x, x_{0}, y, y_{0}, z, z_{0}, \omega\right) \hat{z}$, em um ponto $(x, y, z)$. A fonte e o ponto de observação estão localizados no ar acima de uma terra isotrópica. $\mathrm{Na}$ solução do problema, empregaremos o potencial vetorial de fonte elétrica, $\mathbf{A}$, e a relação:

$$
E=-i \omega\left(\frac{1}{\kappa_{j}^{2}} \nabla(\nabla \cdot A)+A\right), j=0,1
$$

$\kappa_{0}^{2}=\mu_{0} \varepsilon_{0} \omega^{2} ; \kappa_{1}^{2}=\mu_{0} \varepsilon_{0} \omega^{2}-i \mu_{0} \sigma_{1} \omega ; j=0$ representa o ar; $j=1$ representa uma terra homogênea. No caso de uma terra de $n$ camadas, $j=0,1,2, \ldots, n$. Para o dipolo A, podemos expressar o potencial primário por Sampaio (2006):

$$
\begin{gathered}
\mathbf{A}_{P}\left(x, x_{0}, y, y_{0}, z, z_{0}, \omega\right)=C \hat{x} \int_{0}^{\infty} \frac{\lambda}{\alpha_{0}} e^{-\alpha_{0}\left(z-z_{0}\right)} J_{0}(\lambda r) d \lambda, z_{0}<z \leq 0 \\
C=\frac{\mu_{0} I(\omega) d x_{0}}{4 \pi}
\end{gathered}
$$

$\alpha_{0}=\sqrt{\lambda^{2}-\kappa_{0}^{2}}, \mathscr{R}\left(\alpha_{0}\right)>0, r=\sqrt{\left(x-x_{0}\right)^{2}+\left(y-y_{0}\right)^{2}} \mathrm{e}$ $J_{0}(\lambda r)$ é a função de Bessel de primeira espécie, argumento $(\lambda r)$ e ordem zero. Se o dipolo tiver direção $y$, basta trocar o $d x_{0}$ por $d y_{0}$ e $0 \hat{x}$ por $\hat{y}$ nessas equações.

Considere um dispositivo bipolo-bipolo com amplitude bipolar igual a $2 L$ e afastamento entre os centros dos dois bipolos igual a $2(n+1) L, n=1,2,3, \ldots, 10$. O valor do potencial elétrico no domínio da frequência, $\Delta V\left(z_{0}, \omega\right)$, é dado por:

$$
\Delta V\left(z_{0}, \omega\right)=\int_{(2 n+1)}^{(2 n+3)} \int_{-L}^{+L} E_{x}\left(x, x_{0}, y, y_{0}\right) d x
$$

onde $E_{x}$ é o campo elétrico na direção $x$.

As integrais em $x$ e em $x_{0}$ são aplicadas, exclusivamente, sobre a função de Bessel. O resultado dessas integrais produz duas funções auxiliares:

$$
\begin{gathered}
G_{x}(\lambda)=\int_{(2 n+1) L}^{(2 n+3) L} \int_{-L}^{+L} J_{0}\left(\lambda\left(x-x_{0}\right)\right) d x_{0} d x \\
G_{z}(\lambda)=\frac{\partial^{2}}{\partial x^{2}} \int_{(2 n+1) L}^{(2 n+3) L} \int_{-L}^{+L} J_{0}\left(\lambda\left(x-x_{0}\right)\right) d x_{0} d x .
\end{gathered}
$$

A função $G_{x}(\lambda)$ é determinada através de integração numérica. A função $G_{z}(\lambda)$ é determinada aplicando a identidade,

$$
\frac{\partial^{2}}{\partial x^{2}}=-\frac{\partial^{2}}{\partial x \partial x_{0}}
$$

na equação (9) e efetuando as duas integrações por compensação:

$$
G_{z}(\lambda)=J_{0}(2 \lambda n L)+J_{0}(2 \lambda(n+2) L)-2 J_{0}(2 \lambda(n+1) L) .
$$


Como $J_{0}(0)=1, G_{x}(0)=4 L^{2}$ e $G_{z}(0)=0$.

Portanto,

$$
\begin{gathered}
\Delta V\left(z_{0}, \omega\right) \approx-\quad \frac{i \mu_{0} \omega I(\omega)}{4 \pi} \int_{0}^{\infty}\left(\frac{2}{\lambda+\alpha_{1}} G_{x}(\lambda)\right. \\
\left.+\frac{2}{\lambda \kappa_{1}^{2}} G_{z}(\lambda)\right) e^{\lambda z_{0}} \lambda d \lambda,
\end{gathered}
$$

para o caso de um terra homogênea e

$$
\begin{aligned}
\Delta V\left(z_{0}, \omega\right) \approx- & \frac{i \mu_{0} \omega I(\omega)}{4 \pi} \int_{0}^{\infty}\left\{\frac{2}{\lambda+\hat{\alpha}_{1}} G_{x}(\lambda)+\frac{2}{\lambda^{2}} \times\right. \\
& \left.\left(\frac{1}{\lambda+\hat{\alpha}_{1}}+\frac{\sigma_{1} \hat{\beta}_{1}}{\kappa_{1}^{2}}\right) G_{z}(\lambda)\right\} e^{\lambda z_{0}} \lambda d \lambda
\end{aligned}
$$

para o caso de uma terra de $n$ camadas.

Para determinar o potencial no domínio do tempo, efetuamos transformadas inversas de Fourier das Equações 12 e 13,

$$
\Delta \mathscr{V}\left(z_{0}, t\right)=\frac{1}{2 \pi} \int_{-\infty}^{+\infty} \Delta V\left(z_{0}, \omega\right) e^{+i \omega t} d \omega
$$

Conforme indicam as Equações 12 e 13, as transformadas de Fourier dessas equações dependem da formulação da corrente elétrica no domínio da frequência. Assim, com o auxílio da Equação 3 e calculando o integral da Equação 14 obtemos (Mocitaiba 2014):

$$
\begin{aligned}
\Delta \mathscr{V}\left(z_{0}, t\right)= & \frac{1}{2 \pi} \int_{-\infty}^{+\infty}\left\{\frac { - i \mu _ { 0 } \omega I ( \omega ) } { 4 \pi } \left[\int _ { 0 } ^ { \infty } \left(\frac{2}{\lambda+\alpha_{1}} G_{x}(\lambda)\right.\right.\right. \\
& \left.\left.\left.+\frac{2}{\lambda \kappa_{1}^{2}} G_{z}(\lambda)\right) e^{\lambda z_{0}} \lambda d \lambda\right]\right\} e^{i \omega t} d \omega
\end{aligned}
$$

para um terra homogênea e

$$
\begin{aligned}
& \Delta \mathscr{V}\left(z_{0}, t\right)=\quad \frac{-\mu_{0}}{4 \pi} \int_{0}^{\infty}\left\{\sum _ { j = 1 } ^ { \infty } c _ { j } \int _ { - \infty } ^ { + \infty } \left(\omega \cdot \delta\left(\omega-j \omega_{0}\right)-\right.\right. \\
&\left.\omega \cdot \delta\left(\omega+j \omega_{0}\right)\right)\left[\frac{G_{x}(\lambda)}{\lambda+\hat{\alpha}_{1}(\omega, \lambda)}+\right. \\
& \frac{G_{z}(\lambda)}{\lambda^{3}+\lambda^{2} \hat{\alpha}_{1}(\omega, \lambda)}+ \\
&\left.\left.\frac{\sigma_{1} \cdot \hat{\beta}_{1}(\omega, \lambda) \cdot G z(\lambda)}{\lambda^{2} \kappa_{1}^{2}}\right] e^{i \omega t} d \omega\right\} e^{\lambda z_{0}} \lambda d \lambda .
\end{aligned}
$$

\section{Cálculo e Análise dos Dados Teóricos}

Calculamos valores de voltagem com as Equações 16 e 17 , empregando $I_{0}=2,5 \mathrm{~A}$ e os dois valores de $\mathrm{L}$, para os seguintes modelos e níveis:

Modelo homogêneo - $\rho=200 \Omega$.m e níveis 1 e 2 ;

Modelo de duas Camadas $-\rho_{1}=200 \Omega . \mathrm{m}, \rho_{2}=4 \Omega . \mathrm{m}$, espessura da primeira camada igual $100 \mathrm{~m}$ e nível 3;

Modelo de três Camadas - $\rho_{1}=200 \Omega . \mathrm{m}, \rho_{2}=4 \Omega . \mathrm{m}$ e $\rho_{3}=50 \Omega$.m, espessura da primeira camada igual $100 \mathrm{~m}$, espessura da segunda camada igual $200 \mathrm{~m}$ e níveis 4,5 e 6.

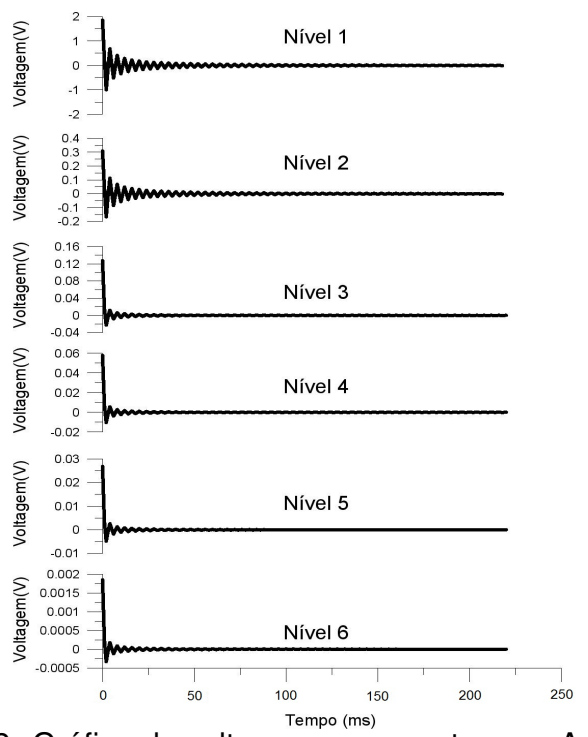

Figure 2: Gráfico da voltagem versus o tempo. Amplitude de bipolo de $50 \mathrm{~m}$ e usando um período fundamental da corrente de 4s.

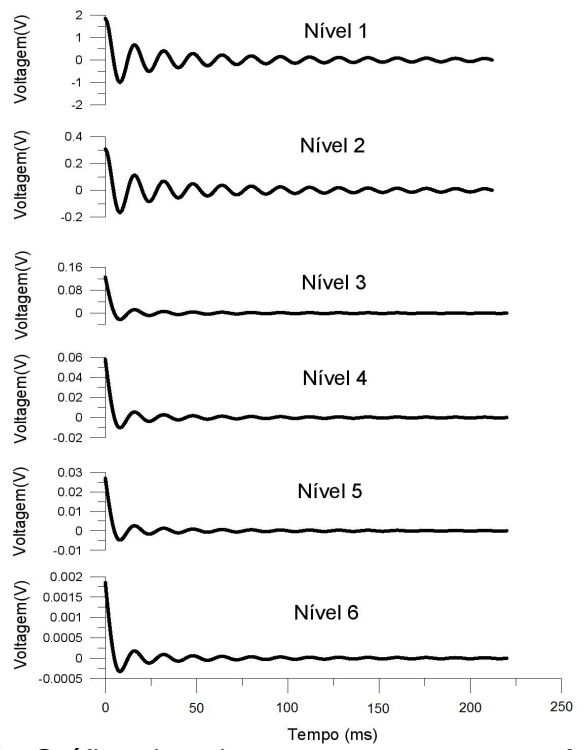

Figure 3: Gráfico da voltagem versus o tempo. Amplitude de bipolo de $50 \mathrm{~m}$ e usando um período fundamental da corrente de $16 \mathrm{~s}$. 
Analisando os gráficos de decaimento da voltagem percebe-se que as curvas de decaimento da tensão exibem elevadas oscilações nos tempos iniciais. Isso é provocado devido ao fenômeno de Gibbs que é um comportamento fortemente oscilatório nos pontos de descontinuidades de uma dada função quando ela é representada por uma série de Fourier.

Comparando as Figuras (2) e (3) percebemos que os valores das voltagens iniciais para cada nível são bem parecidos, indicando que o período fundamental da corrente não influência muito na voltagem calculada no tempo inicial. Comparando o gradiente da curva de decaimento da tensão para os período de $16 \mathrm{~s}$ e $4 \mathrm{~s}$, observa-se que o último apresenta uma queda mais acentuada com relação ao primeiro. Essas análises também se aplicam para as curvas de decaimento da voltagem para 0 afastamentos entre bipolos de $100 \mathrm{~m}$. Com relação à amplitude do bipolo, pode-se concluir que para o caso de $50 \mathrm{~m}$ obteve-se maiores valores de voltagem com relação ao caso de $100 \mathrm{~m}$. Isso acontece porque na situação em que a amplitude de bipolo é de $100 \mathrm{~m}$ os bipolos receptores ficam ainda mais distantes dos bipolos transmissores e, consequentemente, o sinal é mais fraco.

A partir desses valores de $\Delta \mathscr{V}$, calculamos valores de resistividade aparente com o emprego da expressão do arranjo bipolo-bipolo para corrente contínua (Equação 17), os quais estão representados na Tabela 1.

$$
\rho_{a}=\frac{\Delta \mathscr{V}}{I_{0}} 2 \operatorname{Ln}(n+1)(n+2) .
$$

Nos níveis 1 e 2 da referida tabela, os valores de resistividade aparente foram os maiores. Isso já era esperado para esse modelo e esses níveis, pois a resistividade do meio homogêneo foi alta $(\rho=200 \Omega \mathrm{m}$ $\mathrm{m})$. Para o nível 3, o modelo foi de duas camadas $\left(\rho_{1}=\right.$ $200 \Omega \mathrm{m}$ e $\rho_{2}=5 \Omega \mathrm{m}$ ) e a resistividade aparente apresentou um valor médio relativamente alto: $\rho_{a}=125 \Omega \mathrm{m}$. A primeira camada, apesar de menos espessa, exerceu grande influência no valor da resistividade aparente por ser mais resistiva.

Para os níveis 4, 5 e 6, a resistividade aparente apresenta os maiores valores nos níveis 4 e 5 e um valor bem baixo no nível 6 . Esse fato, reforça a análise para o caso do nível 3 , indicando uma considerável influência do alto valor de resistividade da primeira camada nos níveis 4 e 5 . Apenas no nível 6 , a resistividade aparente mapeou a segunda camada, em que $\rho_{2}=5 \mathrm{~m}$. Seria necessário calcular a resistividade aparente para níveis maiores do que 6 de

\begin{tabular}{|c|c|}
\hline Nível & Resisitividade $(\Omega . m)$ \\
\hline 1 & 222 \\
\hline 2 & 192 \\
\hline 3 & 152 \\
\hline 4 & 136 \\
\hline 5 & 124 \\
\hline 6 & 12 \\
\hline
\end{tabular}

Table 1: Tabela com os valores de resistividade aparente para um afastamento entre os dipolos de $50 \mathrm{~m}$ e período de $4 \mathrm{~s}$ e, também, $16 \mathrm{~s}$

\begin{tabular}{|c|c|}
\hline Nível & Resisitividade $(\Omega . m)$ \\
\hline 1 & 141 \\
\hline 2 & 124 \\
\hline 3 & 105 \\
\hline 4 & 96 \\
\hline 5 & 76 \\
\hline 6 & 8 \\
\hline
\end{tabular}

Table 2: Tabela com os valores de resistividade aparente para um afastamento entre os dipolos de $100 \mathrm{~m}$ e período de 4 s e $16 s$

modo a obter informações da terceira camada.

\section{Aquisição e Representação dos Dados Observados}

O trabalho de campo foi realizado na Bacia do Recôncavo (Figura 3.1) na cidade de Esplanada. A linha de aquisição dos dados teve uma orientação NW-SE ( N133 $\left.{ }^{\circ} \mathrm{E}\right)$. De acordo com ( Lima at al.) a região é composta por: Camada 1 - Formação Barreiras de 0 a 100 m de profundidade e $200 \Omega$.m; Camada 2 - Formação Candeias de 100 a $300 \mathrm{~m}$ de profundidade e 3 a $5 \Omega . \mathrm{m}$; Camada 3 Formação Água Grande de 300 a 500 m de profundidade e $50 \Omega$.m; Camada 4 - camada sedimentar de 500 a 1 $\mathrm{km}$ de profundidade e $5 \Omega \mathrm{m}$; Camada 5 - embasamento resistivo.

O sistema usado no trabalho de campo constou de um transmissor modelo VIP 3000 acoplado a um gerador e um receptor modelo ELREC PRO, ambos fabricados pela Iris Instruments. O modo de operação foi no domínio do tempo com uma corrente transmissora tendo forma de onda quadrada.

A aquisição consistiu de quatro sondagens elétricas horizontais (SEH) com 0 arranjo bipolo-bipolo, conjugando os métodos Polarização Elétrica Induzida e Eletrorresistividade (IP/ER) ao longo de uma única picada. Empregamos 6 bipolos receptores para cada bipolo transmissor e um valor máximo de corrente elétrica igual 2,5 A, pois a resistência de contato impossibilitou superar esse valor de corrente. Cada SEH teve um valor para a amplitude de bipolo, $2 L$, e um período, $T$, da onda quadrada, a saber:

SEH-1 - 2L = 50m e T = $4 \mathrm{~s}$;

SEH-2 - $2 \mathrm{~L}=50 \mathrm{~m}$ e $\mathrm{T}=16 \mathrm{~s}$;

SEH-3 $-2 \mathrm{~L}=100 \mathrm{~m}$ e $\mathrm{T}=4 \mathrm{~s}$;

SEH-4 - $2 \mathrm{~L}=100 \mathrm{~m}$ e $\mathrm{T}=16 \mathrm{~s}$;

As duas SEH's com amplitude de bipolo de $50 \mathrm{~m}$ tiveram um total de 15 estações transmissoras em um perfil de $1100 \mathrm{~m}$ e as duas SEH's com amplitude de bipolo de $100 \mathrm{~m}$ tiveram um total de 8 estações transmissoras em um perfil de $1500 \mathrm{~m}$.

\section{Resultados}

A interpretação das medidas de campo foi realizada com base nos dados de cargabilidade aparente $\left(m_{a}\right)$ e resistividade $\left(\rho_{a}\right)$. A Figura (4) exibe as pseudo-seções de resistividade para um afastamento entre os eletrodos de $50 \mathrm{~m}$ e a Figura (5) para um afastamento entre os 
eletrodos de $100 \mathrm{~m}$. As pseudo-seções de cargabilidade para um afastamento entre os eletrodos de $50 \mathrm{~m}$ e $100 \mathrm{~m}$ correspondem, respectivamente, às Figuras (6) e (7).
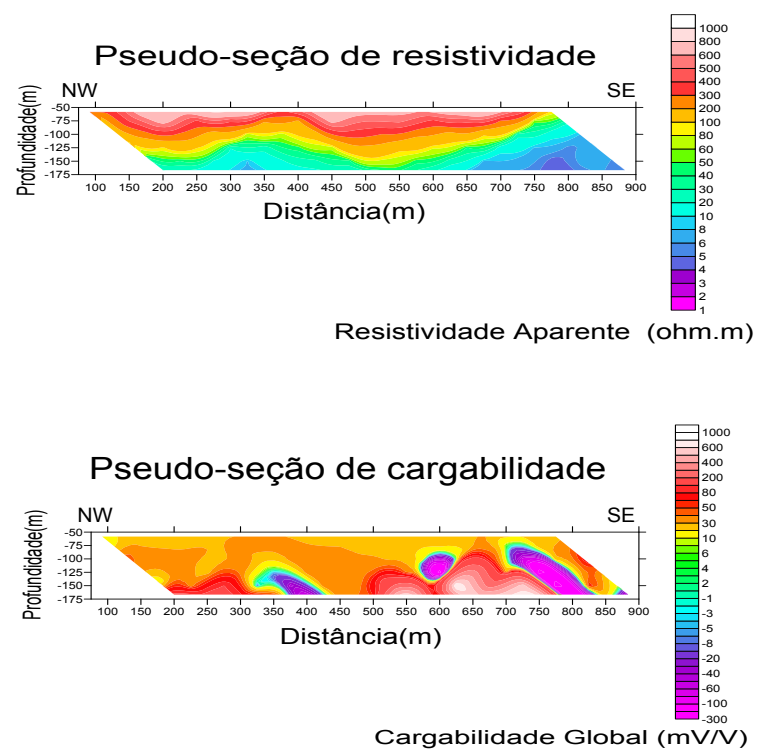

Figure 4: Pseudo-seções de resistividade e cargabilidade aparentes com amplitude de bipolo de $50 \mathrm{~m}$ e afastamento variando de 1 até 6 .
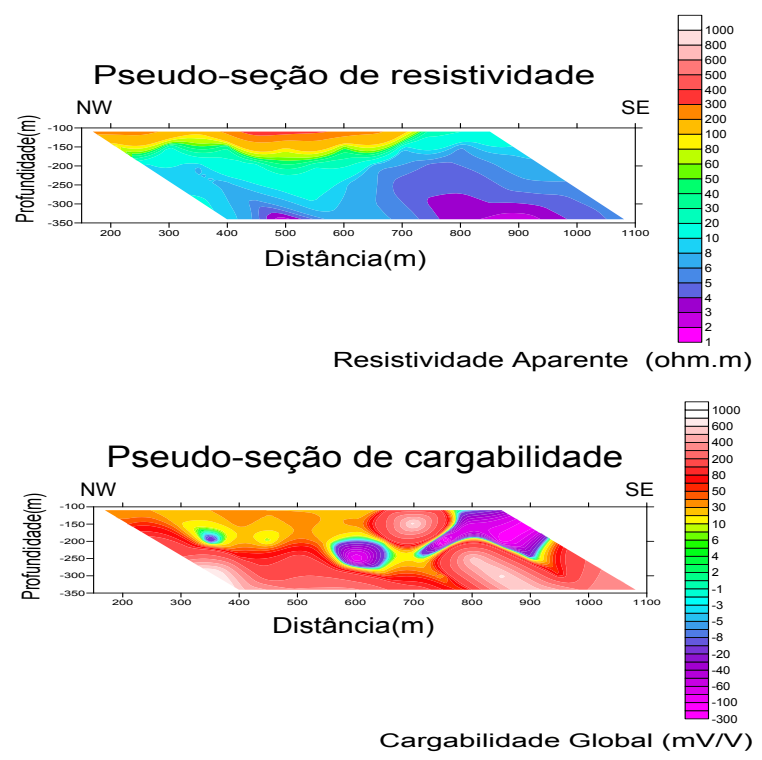

Figure 5: Pseudo-seções de resistividade e cargabilidade aparentes com amplitude de bipolo de $100 \mathrm{~m}$ e afastamento variando de 1 até 6 .

As pseudo-seções de resistividade e cargabilidade aparentes mostradas na Figura 4 representam a geologia de sub-superfície até uma profundidade máxima de cerca de $80 \mathrm{~m}$. As ondulações mostradas nas curvas de iso-contornos de resistividade aparente e cargabilidade aparente refletem efeitos da topografia do terreno, de tal sorte que as depressões nessas curvas correspondem a elevações (morrotes) constituídos da Formação Barreiras.

A imagem da função resistividade aparente se caracteriza por um forte contraste elétrico vertical, com valores de $\rho_{a}$ variando de 5 a $800 \Omega \mathrm{m}$. Nela, estão bem identificadas a cobertura areno-argilosa da Formação Barreiras e a seção superior de folhelhos da Formação Candeias. A parte superior resistiva, com valores de $\rho_{a}$ entre 500 e $1000 \Omega \mathrm{m}$, representa a sequência arenosa da Formação Barreiras, que se estende até profundidades da ordem de $35 \mathrm{~m}$. Subjacente ao pacote resistivo, há uma espessa sequência condutiva, com valores de $\rho_{a}$ entre $5 \Omega \mathrm{m}$ e $60 \Omega \mathrm{m}$, associada a pacotes de folhelhos da Formação Candeias.

$\mathrm{Na}$ imagem de $m_{a}$, os arenitos Barreiras possuem cargabilidades bem baixas, em geral menores que as dos folhelhos subjacentes. É nítida a presença de duas feições tabulares estreitas, com polarizações elétricas bem mais elevadas: uma falha de borda a NE e a outra localizada a 400m de distância para oeste da primeira. Essas anomalias, de intensidades mais fortes que as das formações Barreiras e Candeias, são atribuídas à presença de sulfetos metálicos e argilas precipitadas ao longo de fraturas conjugadas desenvolvidas no topo de zonas de falha.

$\mathrm{Na}$ Figura 5, estão mostradas as pseudo-seções de $\rho_{a}$ e de $m_{a}$ obtidas com o arranjo bipolo-bipolo com amplitude de bipolo entre os eletrodos de $100 \mathrm{~m}$ e afastamento variando de 1 até 6 . Nesse caso, a profundidade máxima de exploração é da ordem de $200 \mathrm{~m}$, e a influência da ondulação topográfica ainda está presente pelo menos na parte superior da seção.

A seção de $\rho_{a}$, em sua parte médio-superior, mostra grande similaridade com a da Figura 4. Todavia, é possível reconhecer um segundo intervalo argiloso mais condutivo, na base da Formação Candeias, definido por um intervalo de variação de $\rho_{a}$ entre 1 e $5 \Omega \mathrm{m}$.

A seção de $m_{a}$ mostra, de modo saliente, a faixa polarizável da zona da falha de borda, assim como um espalhamento lateral da anomalia de IP. Essa anomalia pode ser associada a halos de sulfetos metálicos na parte superior de um reservatório, relacionado à migração de hidrocarbonetos e fluidos hidrotermais ricos em metais dissolvidos, gás sulfídrico e outros, ao longo da falha mencionada.

\section{Discussão e Conclusões}

Com esse trabalho, através do levantamento conjunto de Polarização Induzida e Eletrorresistividade, foi possível identificar e analisar formações da Bacia do Recôncavo. Com base nas pseudo-seções de resistividade foi possível mapear a Formação Barreiras com valores de $\rho_{a}$ entre 500 e $1000 \Omega m$ e a Formação Candeias com valores de $\rho_{a}$ entre $5 \Omega \mathrm{m}$ e $60 \Omega \mathrm{m}$. Essas formações, na região estudada estão associadas a um sistema de falhas.

Os dados de cargabilidade indicaram que a Formação Candeias deve ser mais argilosa, pois essa zona apresentou regiões com valores de cargabilidade acima de $100 \mathrm{mV} / \mathrm{V}$. Já, a Formação Barreiras deve ser composta por arenitos mais secos, pois os valores de cargabilidade não apresentaram grandes variações nessa região e são 
inferiores a $100 \mathrm{mV} / \mathrm{V}$. As zonas com elevados valores de cargabilidade, presentes na Formação Candeias, podem estar associados à presença de halos de sulfetos de ferro, comuns em sedimentos acima de reservatórios de petróleo.

Com os dados dos modelos teóricos da curva de decaimento da voltagem e com os valores de resistividade aparente é possível entender a forma como ela se comporta para os caso de uma terra homogênea e uma terra com 3 camadas. Essas informações foram importantes, pois auxiliaram na interpretação dos dados de cargabilidade e resistividade, indicando, por exemplo, que nos primeiro níveis a existência de elevados valores negativos de cargabilidade podem estar associados a ruídos, tal como o acoplamento eletromagnético.

Por fim, os dados dos modelos teóricos apresentaram coerência com os dado de campo, pois assim como no dado teórico só possível mapear com $\mathrm{n}=6$ a segunda camada, nos dados de campo isso também aconteceu.

\section{Agradecimentos}

Os autores agradecem ao Centro de Pesquisa e Posgraduação em Geofísica da UFBA. Leonardo da Silva Ribeiro Mocitaiba agradece, primeiramente, a Deus e também seus familiares.

\section{Referências}

de Lima, O. A.; Sato, H. K. e Dias, C. A. (2013) Projeto: Uso de métodos elétricos e eletromagnéticos de baixo impacto ambiental no monitoramento da injeção de fluidos em reservatórios de petróleo.

Millett, F. B. (1967) Electromagnetic coupling of collinear dipoles on a uniform half-space, Society of Exploration Geophysicists Mining Geophysics, p. 401.

Mocitaiba L. (2014), Análise da interferência do acoplamento eletromagnético na interpretação de dados de polarização induzida e resistividade, Universidade Federal da Bahia, Salvador (BA), Brasil.

Sampaio, E. (2006) Campo eletromagnético devido a uma linha de dipolos elétricos em um meio condutor, Editora da Universidade Federal da Bahia, Salvador (BA), Brasil.

Wait, J. (1982) Geo-electromagnetism. 1982, Academic, San Diego, CA.

Ward, S. H. (1967) The electromagnetic method, In: Mining Geophysics, vol. II, cap. 2, Society of Exploration Geophysicists, Tulsa, Oklahoma. 